\title{
Rationality and Scientific Method: Paradigm Shift in an Age of Collapse
}

\author{
JOHN MCMURTRY \\ Department of Philosophy \\ University of Guelph
}

\begin{abstract}
This paper explains what has long been missing across domains and levels of analysis: (1) the life-blind inner logic regulating contemporary paradigms of "rationality" and "scientific method"; (2) the reasons why these regulators of thought select for unforeseen consequences of ecological, social and economic collapse; and (3) the principle of life-ground consistency which corrects this systemic incoherence of thought regime.
\end{abstract}

KEYWORDS: academy, collapse, collective choice, full coherence principle, game theory, life and money sequences of value, mechanism, global market, rationality, scientific method, truth

\section{The Nature of the Global Crisis}

The potentially fatal global crisis we face is that humanity's many-faced global industrial regime has led to interconnected and spasmic collapses of the world's life support systems - the air quality, the soil mantles, fresh and ocean waters, species diversity, and stable climate cycles (McMurtry in Miller and Westra, 2002). At the same time, a rising half of the world is destitute, public sectors and services are privatized for profit while a global culture of violence entertainment, waste and military spending escalates. What is not understood at the deepstructural level is that accepted rational and scientific principles have systematically selected towards all of these phenomena.

\section{The Self-Maximizing Principle of Rationality}

At the unexamined core of received models of rational choice is an axiomatic principle which symbolic notation and jargon conceal. It is taken for granted as a formal given. Neoclassical economics, contractarian and decision theory assume it as a first principle, while Marxist critique assumes it as the regulating form of class struggle.

The ruling assumption is the equation of rational choice to consistent self-maximization. Twentieth-century economic, political and moral theory presuppose this first principle as a scientific given. In his canonical A Theory of Justice John Rawls calls it a "principle familiar in social science", and he explicitly includes "to want a larger share for oneself" in the "original position" of choosing principles of justice (Rawls, 1971, p.143). In Morals By Agreement, David Gauthier adds that it applies to morality as well, and means "no upper bound [of the self's appropriation]" (Gauthier, 1986, p. 318). Long before them, Vilfredo Pareto instituted the principle as the producer of "optimality" (ie, the best of all possible worlds") by self-maximizing exchanges in a free market. His 1906 idea is what is now known as "Pareto optimal" (he actually used the term "ophelimity) - that is, an "equilibrium" of affairs in which none can be made better off without another being made worse off. (Pareto, 1971[1906]). None who appeal to the 
principle - and it is now incanted across disciplines - observe that a Pareto-optimal condition is compatible with a few having all assets and most having only debts.

These implications are not discussed within the vast literatures of decision theory, contractarian philosophy and neoclassical economics. Instead, fascination with formal proofs within an idealized exchange of assets bewitches rational choice theory. Human reason is thereby incarcerated within a mind-locked meta-program whose covert value syntax is presupposed apriori.

Deeper analysis discloses five distinct steps set of controlling assumption which are not distinguished or justified: (i) self-maximizing strategies in (ii) conditions of scarcity or conflict over (iii) desired payoffs at (iv) minimum costs for the self to (v) succeed or win. This is the unrecognized meta-program of "rationality" (MPR).

\section{The Invisible Prison versus the Academic Vocation}

The MPR does not regulate as merely a scholastic or scientistic conceit. It is implicitly grounded in evolutionary biology's model of exclusionary competition for survival which is empirically fixed as a ruling dogma. The five regulating steps of thought form an ultimately regulating value set which is taken for granted as the primary framework of natural and social scientific thought as well as formal economic and philosophical reason. Yet nowhere is the fixed given spelled out, critically examined or understood as a regulating value-set. The entire normative structure is, rather, assumed as an axiomatic given not subject to question or modification.

Most disastrously, this ruling value-set is not connected to the collapse of global economic, social and ecological systems which it everywhere leads as the regulating vector (McMurtry, 2002, 2005). The test of the hold of this value program at the highest level of abstraction is to try to find clear exception to its dominion in any ruling decision structure across disciplines, institutions and domains of power.

The instituted protection of academic freedom itself is not free of its rule. The contemporary academic career is structured to seek chooses a self-maximizing career path where many compete for scarce goods to achieve rank and income over others at minimum cost to self. In this way, the MPR has subverted the academy beneath critical notice or correction. The question, "What choice is there?" reveals the depth of the disorder.

The true academic vocation entails an opposite set of regulating principles which are usurped by the MPR sequence at every step. The learning-research vocation is regulated by values in polar opposition throughout: (i) to maximize learning advancement and dissemination by (ii) knowledge sharing without limit for (iii) understanding as value in itself at (iv) any cost of difficulty to (v) develop humanity's more inclusive comprehension of natural and human phenomena.

Revealingly, not even logicians or social scientists identify this systematic opposition of paradigms of rational choice. Instead there is a-priori denial of the conflict. Thus the reflections of high theory adopt the self-maximizing lockstep with no recognition of its totalizing subversion of the constitutional vocation of the academy itself.

The Life-Blind Atomism of the Ruling Paradigm 
At the formal end of high theory and philosophy, "rational choice" consists in countless variations within the theoretical framework of abstract selves seeking maximum payoffs within empty logical self-choice spaces. Consequently, we may observe that in formal studies of "justice" and "moral theory", no life conditions, children, life community, or other species are taken into account (McMurtry, Unequal Freedoms, 1998).

A "postmodern" reaction has occurred over 40 years against universal, rational paradigms - but not because they are life-blind atomisms, but because they are universal. In the postmodern escape from the authority of reason, only "differences" remain. Most postmodern theory itself preconsciously presupposes the ruling order of consumer capitalism as its unseen model (McMurtry in Krishnaswamy and Hawley, 2007). Whatever individuals desire is their right to assert - self-maximizing atomism in a literary form. A few postmodernists like Gilles Deleuze challenge the ruling self-maximizing frame within which all are caught, but for him the liberation can only be by "schizoid deterritorializations" of ubiquitous capitalist flows in "madness escaping control on all sides, and carrying us along" (Deleuze, 1977).

In short, the primarily contesting schools of meaning and value are based on disconnected self-spaces seeking more for their desires with no limit - at one pole, formal, logical and atomic selves, and at the other pole literary, polyvocal and euphoric selves. All pursue self-maximizing sequences decoupled from life means and conditions in accordance with a meta-program none challenges.

Amartya Sen comes closest to moving beyond the limitlessly self-maximizing atoms of economic rationality in his aptly titled article "Rational Fools" (Sen, 1977, 317-44) which points out that "self-maximization" for individual choosers admits of other alternatives than more pecuniary payoffs to oneself. Yet Sen simply blinkers out the money-capitalist system which affords no such alternatives within its mechanisms. When Sen comes to win the Nobel Prize for Economics decades later, it is clear that he still does not think in system terms beyond private choosers. The actually regulating money-capital system remains abstracted out along, and no public-choice alternatives are conceived. As analysis of the text and monumental bibliography of Sen's Nobel Prize Lecture, "The Possibility of Social Choice" (1998), demonstrates, his conception of social choice does not include rational collective agency stewarding the capitalist system itself - for example, by binding life standards built into the market's exchange mechanisms by constitutional public authority, including the global market order (McMurtry, 2002).

All including Sen remain confined within the neoclassical metaphysic of atomic selves and aggregated private preferences. Common life grounds and cooperative agency of decision are thus screened out in principle. Two instituted errors of rational choice and comprehension are thereby locked into ruling conception without notice: (1) A fallacy of division is built into the reigning framework of rationality which cannot be detected from within it (i.e., social choice is fallaciously reduced to the sum of the private choices of the separate individuals constituting the social organization); while simultaneously (2) social life standards to rationally regulate choices to cohere with the enabling rather than the disabling of life systems are thereby blinkered out apriori (i.e., the repressed third principle of rational coherence defined ahead).

Predictably, this atomic-choice framework of self-maximizers continuously generates "the problem of collective choice" which follows necessarily from the fallacious reduction of 
social/collective choice to aggregates of individual preferences alone. "Arrow's paradox" logically demonstrates that no rationally consistent social choices can be derived from the aggregated preferences of individuals (Arrow, Social Choice and Values (1963), and this demonstration becomes the set-point of subsequent understanding. It is assumed throughout that "social choice" can only mean the sum of individual choices, and so "social" and "collective choice" are inferred to be logically impossible or confronted with the impasse of irreducible paradox. Almost 50 years have passed since Arrow published this classical demonstration, with no recognition throughout of fallacies (1) and (2) built into the formal premises which are assumed as given.

The ruling thought-system remains within this metaphysical box from which it cannot escape, but attributes the problem to the formal impossibility of collective choice itself. Since "individual freedom" is assumed to mean only atomic choices exist, any cooperatively governing principle of life-grounded rationality becomes an unthinkable conception within the ruling thought mechanism. Pervasive mystification by closed formal notations masks the foundational erasures locked inside the unexamined first premises.

The original modern-to-contemporary turn of this atomic-choice mechanism is nicely formulated by Frances Edgeworth without the usual mystification of premises. A founder of neoclassical theory, he explains this ruling paradigm of logico-mathematical life-blindness in one crystalline sentence: "The conception of man as a pleasure machine [driven by selfmaximization alone]", he writes, "may justify and facilitate the employment of mechanical terms and mathematical reasoning in social science" (Edgeworth, 1926, p.15, cited by Hodgson, 2000, p.84).

In exquisite core irony, this regulating mechanism is assumed as the defining inner logic of "individual free choice". Few notice that the "individual freedom" of homogeneously selfmaximizing atoms within a mechanical system is a contradiction in terms. Rather, the atomic framework of freedom is geostrategically and methodologically presupposed as the moral basis of free civilization itself - a tacit normative structure called "methodological individualism" or, with complex linguistic mutations, "postmodern difference". Any more broadly and deeply conceived concept of human agency, choice and action is repelled a-priori.

\section{Prisoner's Dilemma: Life-Disconnected Rationality in Paradigm Form}

Perhaps the single most famous paradigm of this metaphysic is "prisoner's dilemma" which has singular pride of place in contemporary formalist philosophy and the social sciences of economics and psychology. In this model, pre-set choices are boxed into one set for two players whose possibility of communicative cooperation is, as always, ruled out a-priori - a formalized metaphor of the structure of alienation from common life interests which rules as the era's framework of value decision.

The dilemma which the private choosers confront is whether to "defect" (confess), or not. If one confesses and the other remains silent, the one who turns coat goes free, and the one who remains in solidarity gets ten years. If they turn on each other for self benefit, they get five years. If both remain silent, they each get a light sentence of two years or less. What does one rationally do as a self-maximizer? That is the only dilemma.

Once released into academic currency, cascades of disputes were generated within its 
choice spaces void of all life substance and relations. "Reiterated", "n-person" and "free rider" variations fill journals and books across specialties and disciplines. All conform to one value syntax. Co-operative unities of persons deciding from common grounds of life interests are ruled out in principle. Thus the civil commons infrastructures on which society is based - social constructs which enable universal access to life goods, for example, protected air to breathe - are abstracted away a-priori by exclusively atomic aggregates of decision.

On the wider level of the real world, collapse of social and natural life infrastructures follow from this self-maximizing atomic "rationality" everywhere "self-regulating" with no regulating collective governance except by the "invisible hand" : which is, in turn, assumed by this structure of rationality to generate Pareto-optimal outcomes for all. In a poignant but unremarked symbolization of the wider social conditions within which the paradigm of prisoner's dilemma is presented, all the relevant coordinates of the dilemma are blanked out what the criminal accusation is, whether either party is guilty or innocent, the justification or conditions of being prison-caged itself, and anything to do with examining the human purposes and life values by which a sane humanity or society governs itself. Erased Kafka-like from the story, only atomically self-serving preferences rule as "rational".

In reality, there are self-evident life values demanding public clean water, an unpolluted environment, and other collective goods at every level of human existence. But none can be decided for from within this locked frame of choice without problems of "free riders", "paradoxes of aggregate preference" and other doctrinal blind alleys. This cul de sac of the ruling paradigm of atomic self-maximizers is, however, nowhere counted against the paradigm itself. Instead, the paradigm's own dead end is projected onto what it cannot accommodate or explain, and is ordained "the problem of collective choice". This problem, in turn, is assumed to explain why collective agency is disproved as a coherent possibility.

In this way, the very failure of the dominant model of rationality reinforces the assumption that it alone is logical - a familiar closed circle of doctrine which may be called "system mind-lock". Accordingly, it continues to be assumed that rationality is self-maximizing atoms seeking ever more for themselves as the sole ground of epistemic rationality, realistic choice and individual freedom at the same time.

The inner meta-program is crystallized within the 10 presuppositions of game theory and rational preference which regulate this ruling paradigm of rational choice. They are not made explicit, but can be laid bare from game theory and global-market choice spaces by what Immanual Kant called "transcendental deduction". That is, they are deducible as necessary conditions of the experienced regulation of these phenomena.

(1) All agents seek only to maximize their own preferences.

(2) Each agent's preference-object is fixed.

(3) One's competitors in the game are not subject to choice.

(4) One can appeal to no standard of justice or right external to the game-structure.

(5) Each player's position is preordained independent of moral desert or life need;

(6) All choices and outcomes are prescribed in advance.

(7) The preference order of payoffs and losses is inalterable.

(8) No concern for anyone else's interests can influence choice save by its impact on one's own payoffs or losses. 
(9) No decision may be related to any relationship or tie of the players beyond the game.

(10) No payoff received relates to life contribution or need.

Together these regulating presuppositions define a general axiological syntax by which the global market, the military institution defending and advancing it, and other instituted orders of prescription are governed on the ground as well as, more purely, on the level of theoretical representations. One may put a proletarian worker or a tenured philosopher into the choice space of atomic agent, and the syntax remains constant. All are expected to self-maximize; to prefer always more to less money; to compete in the market with whomever desires what one also wants; to know that there is no standard of value that can overrule the rules of the game; to accept that one is born into it and goes where one is assigned with no moral claims beyond its order; to accept the options and outcomes as they are set to maximize one's own position; to hold one's course of choice consistently to succeed; to not worry about others or what is not your assigned job; to stick to one's place within the given order; and to not expect that any who are better positioned have contributed more to human life or need what they have.

This is the regulating framework of official culture that is not penetrated or discussed. It regulates academic careerists as well as business executives. More deeply, it is the meta-program of reason itself which elaborates the original formula with which we began - an implicit ten commandments of how all are assumed to think and live as "rational". At the heart of this lifeblind meta system lies received scientific method.

\section{From the Fallacy of Scientific Neutrality to Global Industrial Science}

Almost all readers will be aware of the long institutionalised claim of the sciences to be "value neutral". This is not only a long-standing claim of representatives of the physical sciences, but of mainstream imitators in the social sciences. How often, for the most presumptuous example, have we heard "economics is neutral" and "economists merely provide options for others to choose among"?

The standard assertion of the value neutrality of science is seen to be illusory in every case as soon as we recognise the normative principles in terms of which scientists consistently judge findings of fact and hypotheses of their explanation. Pro and con principles of validity and invalidity guide every judgement and conclusion without whose regulating values science would cease to exist. Laboratory scientists, for example, are governed by absolute values of peerverifiable evidence and experimental protocol for their work to qualify as scientifically "valid". At the same time, exact identification of confirmably invariant sequences of phenomena distinguish "good science" from "bad science". However much they may be habitually and mistakenly assumed as value neutral, these are in fact strict norms. That is, they prescribe exact forms of behaviour which can be transgressed; and are in fact what all scientists are obliged to comply with if their work is to be recognised as acceptable rather than false and blameworthy. They constitute the underlying scientific ethic which is now given.

That science is a value system cannot be denied without self-contradiction. Hence we may speak of the "value-neutral fallacy" that wrongly supposes science is a value free enterprise. Yet what is fatally missing from these regulating values is any principle of coherence with the conditions of the world's own conditions of reproduction. These premises can be ignored until feedback consequences from scientific technology's interventions in the world 
began to distort the life support systems upon which humanity depends.

Consistency with these unseen first premises is thus obligatory to maintain the coherence of scientific method. Without any scientific standard to ensure against ever more ingeniously constructed ways to deracinate human and planetary life in the name of "rationalization", "science" and "development", this deep-structural inconsistency with life support systems becomes fanatic devastation in service to money-capital interests and commodification. The failure of science and reason to rule out this baseline incoherence has, however, remained analytically invisible within the unexamined premises of the reigning paradigm. Scientific reason must take account of the relevant facts it now blocks out by submersion in this external metaprogram. A higher level of scientific integrity, evidential consistency and predictive capacity is required to overcome the set-points of life-blindness.

The general resolution follows from the general problem. Scientific rationality requires more inclusively coherent set-points of comprehension. The essential and unrecognized flaw has been that scientific method has no internal regulator of consistency with life support systems. What distinguishes scientific method - openness to disconfirming empirical data and testable resolution of anomalous outcomes - has been thereby invisibly abdicated where these standards count most. The primary reason that the dominantly funded sciences are indifferent to whether scientific research and technology are consistent with or contradictory to human and ecological life systems is that this dominant paradigm to which it is servant is structurally biased towards a sectarian goal - whether science can "add value" or be "brought to market" to maximize revenues and powers for extrinsic ruling interests. Since these special interests externalize all costs except to themselves, what is cumulatively disastrous for human and ecological life systems does not factor into the scientific accounts of those serving them.

This is why research for high-end destructive weapons, mass consumables which depredate human health, and oil-consuming, polluting and carcinogenic commodities and additives and life-blind mega projects can all multiply in deleterious effects with no feedback loop of scientific recognition or principled correction of them. Contra-indicative phenomena on life-system levels are simply blocked out. If informed witness or whistle-blowers call them to attention, the ill effects are denied and critics are denounced.

Beneath these phenomena of conflict, prior norms of independent testing and precautionary standards are overridden by state and corporate "risk-benefit" formulae and policy demands in which the ruling criterion of scientific worth has become maximization of external advantage in private-sector commodity competition. "Peer-reviewed science" within this mechanism becomes only a cudgel to silence independent criticism. In the background, pervasive invocations of "the necessity to compete in the global market" drown out and marginalize voices of opposition across borders.

The anti-scientific pattern at work to silence evidence of ill consequences is simultaneously taboo to identify within mass-media corporate ad vehicles, public bureaucracies subserved to corporate state parties, and captive university administrations which have themselves led in seeking private corporate funding of research capacities to make up for government defunding. For example, Canada's university presidents combined 50-50 with corporate executives in the Corporate Higher Education Forum argued in 1980's reports that universities could solve their problems of government underfunding by serving industry with 
proprietary research programs and curricula tuned to their external agenda (Buchbinder and Newson, 1991).

The idea that "bringing research to market" must take account of society's known life support systems before it is valid or publicly approved is thus inconceivable within this captive culture of research and science. "Defense research" that systematically threatens civilian lives and "life sciences" that are life-blind follow as normalized oxymorons. Coherently objective distinction between life-enabling and life-disabling methods, processes and products is inconceivable within the ruling thought regime. Not even a criterion of "knowledge" substantiates pervasive claims of "the knowledge economy" which is systematically blind to lifeand-death distinctions.

"Science in the public interest", once sovereign within universities and public-sectors, has in these many ways been usurped, and researchers holding to it made persona non grata. Under slogans like "public-private" and "university-corporation partnerships", the research and learning agenda are made accountable to strategic proprietary positions of local and global market advantage to which the common interest is absurdly equated. Science which stands for or is validly coherent with life support systems is conversely selected out as "unaffordable", "inflexible" and "non-competitive".

The resolution is straightforward. Either scientific research and enterprises are made consistent with life-system standards, as defined ahead in True versus False Rationality and Science, or they are reduced to servo-mechanisms of life-blind external power and command.

\section{Collapse: Blocking Out the Cause-Effect Mechanism}

The results of the ruling meta-program in which science is merely an instrument is ignored lifedestructive impacts accumulating towards catastrophic proportions which are not connected back to their structural cause. Thus Professor Jared Diamond's best-selling book, Collapse which names the prospective catastrophe, duly blinkers out the system disorder itself (Diamond, 2005). The International Forum on Globalization (IFG) consisting of academic researchers, economists, non-governmental administrators and writers representing over 40 organisations from 20 countries recognises the cumulative collapse earlier and more systematically than Diamond, but also fails to track the causal mechanism of the reported phenomena of radical life-system disorder. "The pattern of recent years has been - - massive economic breakdown in some countries, growing unemployment and dislocation in all regions, direct assaults on environmental and labor conditions, loss of wilderness and biodiversity, massive population shifts - - conversion of [water, forests and soil] to luxury commodities - - increased hunger, landlessness, homelessness - - and insecure food supplies, lower food quality and contaminated foods as secondary outcomes". IFG reported these phenomena a decade ago in the 1998 Sienna Declaration, but the interlocked policy and funding regime selecting for the systemic disaster remains taboo to think or name.

While these cascading losses of life bases are studied in unconnected silos of very detailed information, their underlying pattern across domains is not linked across phenomena or to their upstream causal mechanism. Rather, it is screened out a-priori by screening out the ill effects as "externalities", a category coined by neoclassical economics which erases these causal connections a-priori. Only monetary quantities of priced exchanges compute within its stripped- 
down thought system (Mirowski, 2000).

The wider and deeper pattern becomes increasingly evident, but is seldom connected across domains of expertise, and least of all back to the common system-deciding mechanism. Rather, the system is assumed as an autonomous necessity independent of human decisions. A ruling megamachine is presupposed by both creatures and critics to which life-system coordinates are alien. Let us succinctly identify the global pattern that is not decoded.

Ongoing collapses of aquifer reserves, ocean fishstocks, coral reefs, forest habitat, primary arable land, nutritional contents of processed foods, tolerably quiet zones, and songbird numbers all proceed together with no connected explanation. Although almost everything which lives and which human life requires is thereby endangered, the "rational and scientific economic decisions" selecting for the collapse are blinkered out of causal explanations. As the deepening and widening profile of degradation of life support bases becomes undeniably manifest in biophysical reality, the presupposed meta program demonstrates ever more systematic incoherence with planetary life reproduction itself.

A far-reaching decline in the vocational capacities of the human species goes simultaneously unidentified within this downward momentum. Fewer and fewer stable livelihoods remain to the next generation to provide what they and the world most of all need work of value to life and life fabrics, as distinguished from private manufacture and sale for maximum money returns. Beneath notice the dominant demand of making and selling commodities which do not enable but disable life by their processes, products and wastes reverses human evolution itself. Step by step the reigning choice mechanism undoes the deepest bonds of humanity and social cohesion - to do meaningful work of life value for others - at the same time as it as it depredates ecological life systems and the life-means telos of human production. The unseen ontological bridge of humanity across the self-other divide is blocked and distorted at every level. To the inner logic of this inhuman man-made mechanism we now turn to disclose the ultimate track-switch of individual and social choice which has been overlooked.

\section{Decoding Our Condition: True versus False Rationality and Science}

Rationality of any kind must be consistent with its objective to qualify as rational. This is well known. The unseen problem is that true and false forms are not distinguished. Distinction between what consistently enables and what systematically disables life fabrics is not made. Any life despoiling plans, processes and cycles at all may be "rational and scientific" so long as more non-living quantities go to self-maximizing selves. This is the inner logic of idolatry that has long ruled the human condition.

Modern rationality means merely consistent choice sequences to meet this objective, whatever form it may take. Life coordinates and standards are excluded from account a-priori. In this epoch, the metric of money units - homogeneously additive and life indifferent - have become the lingua franca of this meta program's choice space. So convenient is its simple, fungible uniformity of quantification that most economists and social scientists cannot think without it. Self-maximizing exchanges of money-values substituting for all upon which life systems depend have thus become the ruling lock-step of our lot.

Money-count rationality rules even the everyday life of the street. Who does not prefer 
more to less of it at every choice turn? But it invisibly generates absurd and fatal results at the system level. For example, per capita income and citizen well-being are equated although they may be contradictory in meaning - one referring to gross national money transactions (e.g., by more junkfood sold) and one referring to life value enjoyed (e.g., by less junkfood bought). There are countless such examples of absurd consequences of this form of "rational" calculus. All begins with the a-priori first premise that money exchanges are "goods" in what one gets by them, never "bads" - although these "goods" increasingly disable human and planetary life capacities in fact.

The internal contradiction here may be laid bare by identifying the true and false sequences of rationality which may be followed, but which are not distinguished. Both sequences of value are internally consistent, but only one is consistent with gaining rather than losing life and life value. The life sequence of value (Life | Means of Life | More Life, or $L / M$-of-L $/ L^{l}$ ) is alone rational at the life-system level. It always deploys means of life to yield better life: and better life rather than worse life is the only rational sovereign objective of human beings. This is a principle which is self-evident, but decoupled from.

On the other hand, the money sequence of value ( $\$ / L$ as $\left.M / \$^{1,}\right)$ deploys life and lives as its means - including the possessor of it - and seeks only to maximize money possession. This is naively called "value added" in the currently ruling paradigm. Yet it is ultimately an irrational choice sequence, however consistent and efficient in means used to maximize its end - because it leaves all life requirements out of account. It eventually leads, as it now has, to the collapse of life support systems through generational time.

Observe that it is only when we widen the framework of consistency with fact and other statements to consistency with life requirements that we lay bare the irrationality of the money sequence of choice. However internally consistent it may be, it is a-priori life-blind. Its derangement can be understood only when scientific rationality is opened to wider parameters of accountability. This is the paradigm shift required to steer out of cumulative life system collapse, and its rational regulator is consistency with, rather than blindness to, life support systems.

As we come to understand that truth is always a process of more coherently inclusive taking into account, and life coordinates are what must always be taken into account to be consistent with our terrestrial condition, reason awakes. It recognizes that sound logic, science and rational choice must meet three criteria of truth:

(1) consistency of premises with evidence;

(2) consistency of inferences with premises; and

(3) consistency of objectives with life support systems.

These criteria together constitute the full coherence principle. (1) and/or (2) are the traditional standards of science and logic respectively - although logic and philosophy have widely abandoned empirical bases in self-referential conceptual systems. At the same time, science and its physics paradigm have excised philosophy, or have imagined like Stephen Hawking that they are doing philosophy within nano-second referents of a conjectured universe beginning. Natural philosophy and philosophy have in these ways separated - except at the full coherence level to which neither has yet evolved. Principle (3) of rationality has, at best, been only inchoately implicit in the most advanced works of human reason. In general, the countless contemporary models of philosophical and scientific rationality are simply blind to principle (3). 
They are indifferent to whether their means and ends disable rather than enable life support systems, and in this lies their meta program of rootless disaster.

No-one can coherently deny any one of these three requirements of rational thinking. It cannot be rational to ignore or flout empirical evidence, to be inconsistent in claim, or to follow a life-blind purpose. Yet reason's three-fold requirement of consistency with facts and other statements and universal life requirements has been stripped of its baseline criterion.

In this light, we might observe that on the far side of scientific method, postmodernism and feminist epistemology deplore lock-step closures to difference and ways of seeing, but derive their own persuasive power from the underlying erasure of principle (3) whose universal life base remains repressed across contemporary thought systems.

\section{Life Support Systems as Ground of Reason}

When "two thirds of the natural machinery that supports life on Earth has already been degraded" (as concluded in a report of 1,360 scientists launched at the Royal Society of London in April 2005), human civilization palpably meets a turning point of re-grounding its system deciders, or destroying the planetary life-host. Yet even in world-expert recognition of a resource draw-down crisis, the problem is posed as merely "degraded machinery". That infinitely interconnected life webs and support systems are quite the opposite of machinery - they cannot be reversed - does not compute even to the renowned scientists sounding a global alarm. Recognition stays within the reversible mechanical model even as this model structures towards collapse of our terrestrial roots.

At the most general level of determining mechanism, our ruling syntax of thought steers for ill-being as much as well-being of our life-systems. It does this by blinkering out the difference between them. This is why even the concept of human need or life necessity - that without which life capacity is always reduced - does not exist in received scientific or philosophical lexicons or their measures. In ruling economic doctrine, need and greed cannot be distinguished because only the money-demand backing either counts. As the life-blind mechanism becomes increasingly ruinous in unintended effects by money-sequence selection of scientific technologies indifferent to external despoliations and wastes, the modern evolutionary strategy of the species begins to emerge beneath the radar of official science and culture as a long-tern catastrophic failure.

As human and natural life and life support systems are drawn down by what is absurdly declared as "economic efficiency" while producing the most wastes in history, concerns arise from within established quarters about overstepping natural bounds. Yet still there is no connection back to the meta-program selecting for the life-system depredation. Thus analyses such as The Limits to Growth (Club of Rome, 1972), and Our Common Future (World Commission of the Environment and Development, 1986) recognize a general problem of system overreach, but do not link analysis back to the life-blind selectors at work.

A much-favored explanation is, instead, world population increases. Yet because global population increases are abstracted from the conditions of mass poverty and industrializing conditions producing them wherever they occur (with negative endogenous population growth in welfare-state conditions), the deeper cause-effect relationships are blocked against. Only selected aggregate effects are seen. The exponential growth of the money sequence commandeering all 
else is not related to the correspondingly exponential growth of impoverished populations. Lifeblind principles of rational decision both lead and block out the underlying causal mechanism.

What is to be done at the scientific level? What models of science and rationality now lack is coherence with the life support systems upon which all depend, and what the third principle of scientific rationality requires. Without consistency of science's objectives and means with the laws of reproduction of human life and life conditions, cumulative deracination of the conditions of human existence is predictable in principle. Yet block against such recognition recurs at a second-order level. Public authority, it is believed, must not "interfere with market freedom and efficiency". At the same time, "the state's neutrality between conceptions of the good" disqualifies higher life standards to which market activities and scientific technology must be accountable to achieve human and planetary well-being. Again, the reigning framework of atomic choice locks out life-grounded conception

As long as discourses of rationality, science and policy are closed to what does not fit their ruling paradigm, global culture across divisions joins hands in a thought regime which cannot distinguish between ways of life and death.

\section{Reclaiming Rationality and Scientific Method from the Global Market Maw}

Scientific method, like rationality, is a set of standards intended to rule out biases of method, blocking against evidence, and the prejudices of powerful interests. It is meant to discover the truth of hypotheses in explaining human and natural phenomena with capacity to recognise and respond to contra-indicative evidence. Funded science has, however, been increasingly structured to do the opposite (McMurtry 2000, Press and Washburn 2000, Turk 2000).

The dangers are built into the nature of the regime. When research and learning are valued not for more inclusively coherent understanding, but for "competitiveness in the global market", deep-structural conflicts of interest arise in principle. Research obeying the dominant regime is biased to serve external and private market demand, or it is selected out. Consider here a flagship indicator, the Canadian AgriFood Research Council's 1997-2002 National Strategy, a directive distributed by university research offices to researchers before they apply for funds. "Increasing competition for research funding", it warns, "will demand that Canada identifies its research strength and capabilities to focus on those areas with highest value and return on investment. Prorities for applied research are set by the marketplace via partnerships e.g., industry funds research that fits their priorities. Augmented private sector participation in research priority setting will ensure scientists have access to the appropriate market signals, are aware of the technology requirements of industry, and focus their research appropriately".

As the generic language of this document indicates, this is a government policy template for public funding and higher research prescribed across jurisdictions. For example, subsequent to this directive, introduction of a "research integrity" condition to funding of medical research by Dr. Robert McMurtry within the federal Medical Research Council was agreed to by Council members, but annulled without justification by the ruling centre of government. "On two occasions", he reports in confirming correspondence, "the MRC board moved and passed a motion that Pharma contributions of $\$ 60$ million per year should be given with no strings attached i.e. added to the general pool of grant funds and not be adjudicated by committees with industry representatives. On both occasions, the motion died even though the standing committee 
on health also voted the same way." "As to the assassin", he adds, "it is not possible to say with certainty because it is done behind closed doors from the centre, likely the PMO "(May 10, 2008).

Public funding of higher research in such ways has not only priorized commercial research, but has discriminated against non-commercial research whose funds are increasingly appropriated to serve external and private market interests. Thus without notice, research into collective goods and bads are defunded and marginalized. They do not attract private corporate sponsors or partners. The most important scientific and rational innovations are thereby erased from possibility at research as well as public policy levels - from unpatented low-cost pharmaceuticals to mandatory collective recycling systems to convenient alternatives to privateauto transit to national public-water and energy programs for ecological purefication, extraction and processing.

As researchers and graduates are employed and more highly paid to sell, manage or research private commercial products and profit - and increasingly universities are structured to do so - the academy ends up serving these special interests and their pollutive-waste externalities rather than critical understanding and problem-solving research. Unnoticed within a global market evangelism of public policy and budget fiats by state and academic administrations, the fact that advancement and dissemination of knowledge for the common life interest is in principle an opposite vocation is locked out of comprehension.

There have been growing debates around this nest of issues - whether pharmaceutical and medical corporations have hijacked medical science as a servant function to their own profit agenda, whether students conceive their education as merely a middle term for selling themselves at a higher price, and whether higher education has become a handmaiden and patent player of corporate money sequencing with universities as their publicly funded resource. The critical literature here has grown, but lacks any clear common ground or conception of the regulators of validity required. Consistency of objectives with life support systems remains absent as a generic standard.

One underlying question to any scientific enterprise reveals its life-system coherence or incoherence. Does the scientific objective and method include consistency with life systems by its proposed advance? This question is not asked. No regulating standard to select for enabling rather than disabling human and environmental life systems is yet built into the scientific protocol of any research domain.

Predictably, the structuring of scientific method and research is as blind to harm to life and life means as the funding interests in control of the agenda. Thus scientific and technological enterprises exclude life costs and life support services from account because they are external to the self-serving market exchanges, while at the same time these agents jealously guard their unaccountability to any common interest as their "market/academic freedom".

That the public is paying the basic research costs for goals and methods to serve private interests which lead systemically pernicious effects on the biosphere is not an issue that is discussed. So long as no scientific standard makes research goals and technologies accountable to their downstream effects, then they are funded merely for maximally profitable outcomes to external paymasters. Virtually any life-destructive project proceeds as scientifically valid research for biological weapons, genetic piracy, suicide seeds, assembly-line caging and 
mutilation of animals, chemically adulterated and genetically contaminated edibles, and so on. The costs and losses to human, animal and ecological life are excluded.

Who rebels? The question again discloses the depth of the problem. In the macrocosm as well as microcosm, the scientists and scientific bodies sustaining this regime receive the lion's share of wealth for research while organic research at every level is cut out, and "managed risk" is the corporate-government rationale for overriding any hazards that are undeniable. Since systemic life depredations following from the invisible-hand regime are not taken into account by the nature of the system, it is out of order to question the ruling purpose within a religious-like system worship. A macro pattern of cooked science - research tailored to suit funders' external, unscientific interests - thus develops which is both undetected and increasingly enriched within the dominant regime. No questions are allowed on pain of non-funding. The formerly reigning standard that "publicly funded science must serve the public interest" is displaced by the assumption that what is good for market gains is good for the public interest - an equation of doctrine whose totalizing prescription is the essential fanaticism of this ruling thought regime.

The elementary standard to ensure against such instituted biases at the front end of research is well known, but suspended in the global market era. It is the rule of scientific integrity, and its principled base is that any science in which the funder or partner has a financial stake in the outcome of the research is invalid in principle. Only results by independent scientific tests qualify as impartial science. The abandonment of this standard of scientific integrity by government agencies and university scientists in Canada and elsewhere, as reported earlier, has followed an historical pattern. Abdication of independent government testing of commercial products and the simultaneous commercialization of research in universities has moved in lock-step with secretly negotiated, electorally unaccountable and treaty-imposed "free trade agreements" from 1988 on (McMurtry, 1998). This twin-policy pattern is now well known to informed observers. Yet it continues to be demanded across borders by corporate governments to "compete in the global market", and - at the logical level tracked in this article - is a pure-type expression of the myopic meta program of rationality (MPR) defined at the outset of this study.

Until the rule of scientific integrity is restored, research which is not compliant with external interests of money sequencing is purged of funding support. Since non-funding by external agencies and corporate partners is increasingly a sentence of death to higher research upon which even graduate supervision now depends, a pervasive threat is silently implicit in the reigning order - go along, or forfeit academic/scientific career. This regime suppresses critical reason and science more effectively than direct censorship by the medieval church. Its invisible pattern may be confirmed by searching for any well-funded contemporary research that does not conform.

The very nature of rationality - to inquire and observe without external bias and selection of results to suit private gain - is in these ways usurped beneath notice. On the ground, the slowmotion depredation and collapse of organic, ecological and social life infrastructures which remain without connection to the man-made causal mechanism are predictable outcomes of the life-blind thought regime.

Beyond Cooked Science: The Paradigm Shift

The first standard required of scientific rationality is independence from powerful external 
interests for which science is now tailor-made to get funding. The "research integrity principle" defined above provides the first standard to rule out this primary determinant of cooked science.

The second standard is more complex. Scientific method must take account of contraindicative evidence which is blocked against by closure of predictive range - for example, genetic engineering by insertions of dominant-gene vehicles into organisms with no testing on generational effects within or beyond the organism. Rational and scientific forethought is obliged to include a wider scope of evidential forethought to be scientific, but is not. It is here that the predictive capacity of science is tested at the level which counts most - anticipated and tracked effects on human life support systems through generational time. This generic version of the precautionary principle is what is lacking at the core of science's vaunted powers to predict.

As we have seen, subordination to the rule of reduced costs and increased revenues for private money-sequences militates against this predictive capacity in principle. Whatever adds costs without guaranteeing more revenues or powers to funders is ruled out. Since only money costs count within this value calculus, all other costs are blinkered out a-priori. This purblind partiality has led to a world where even the destabilization of the earth's cycles is excluded from all accounts through every stage of its causation.

The resolution to this reigning ignorance again follows from the full coherence principle. To be accepted as valid, research objectives and designs are obliged to take into account potential ill-effects on life and life support systems as a condition of their scientific funding or subsidization. This regulating condition resembles the physician's long-tested principle of "no harm" applied to science in general. More directly, it is intrinsically required by scientific logic itself which is obliged by its internal norms to take account of all relevant evidence to its objectives.

In this way, the deep coherence principle rules out what has masqueraded as "science", but structurally prejudiced to the gains of special interests alone: for example, chemical engineering of toxic inputs across the production-consumption cycle without adequate scientific standards of disease prevention, for-profit interventions in cancers and illnesses disasters following from such inputs whose manufactured determinants are neither investigated nor controlled, and so on. All of these harm-cycle phenomena continue and increase because their consequences have not been tested for in advance. Hazardous, morbid and fatal effects systemically increase so long as scientific corrective feedback loops are not built in.

The problem here is not only selected-fact programs masked as science, but rising epidemics of dispossession, disease and death to countless people and life conditions by the forces which this pseudo-science serves and advances (e.g., by commodity carcinogens to reduce costs), whose victims may then be further exploited as new markets (e.g., as cancer victims requiring expensive pharmaceuticals, equipments and treatments). This vicious and widening cycle cumulatively invades all that exists - market failures propelling new market opportunities as a primary growth dynamic (McMurtry, 1998,1999).

We might pause here to ask ourselves where this pattern does not now advance as a selfreinforcing cycle. There is a mixed picture at the public policy end. The European Union has been alone in directing that "where there are reasonable grounds for concern for the possibility of adverse effects, but scientific uncertainty persists, priority will be given to human health and the environment without having to wait until the reality and seriousness of those adverse effects 
become fully apparent". It has accordingly drafted comprehensive environment laws to regulate against toxic chemicals (legislated in 2004 and in force from 2007) and an Accounts Modernization Directive (2006) which requires that all publicly traded European firms must include future environmental liabilities in their accounting costs and potential future profits" (Ferrie, 2008) - initiatives which are fiercely opposed by the U.S. via diplomatic pressures, up to 15000 lobbysists, and blocking instruments like the World Trade Organization (with Canada following in line like a branchplant state). On the global front of deadly disease, "cancer incidence continues to grow in parallel with gross national product and industrialization, but the obvious explanation for this phenomenon - environmental pollution, chemical and radioactive, is ignored [including by the World Health Organization]" and "victims are blamed for their lifestyles" (Katz, 2008, p. 12). The "commercial threat must at all costs be averted", and so the conflict of interest between health institutions like the WHO and powerful cancer-research organizations/charities, on the one hand, and the multi-billion-dollar atomic, pharmaceutical, medical equipment and military industries to which they are linked (Epstein, 2005) has been fatal for countless people across the world.

Within the unseen moving spring of this transnational science-industrial complex, the best-trained experts within specialist domains are not required to know what they are doing any more than external funders and stock-holders. This situation of systemic ignorance is, in turn, blocked out by exclusive attention to the mounting ill-effects decoupled from their regulating mechanism even at the highest levels of critical alarm - as in Al Gore's celebrated Inconvenient Truth, and - more generally - virtually all science media programs lamenting the perceptible collapse of other species and the rise of industrial-disease epidemics (themselves not connected).

The response from within the scientific community to these eco-homicidal linkages is to blame "politics" which scientists are above being concerned with, or self-justification at a disingenuous and symptomatic level - "this is the only way science and scientists can get funded". Yet these externalizations of responsibility merely echo higher powers and highlight the necessity to recognize the deep disorder at the scientific level. Most of the funds for scientific research and training are paid from public budgets from educational training through massive research subsidies to campus facilities of universities. Scientists and scientific communities are the central managers of science curricula, methods, and protocols within these institutions, as well as highly placed inside governments, policy-making bodies and classrooms. Scientists of all kinds are in a better position to rule out science cooked for external demands than anyone else: while researchers in philosophy and the humanities are as able and obliged to be coherently rational rather than life-blind.

Science or rationality without standards to ensure objectives and means consistent with global life support systems is in the end neither rationality nor science. Insofar as truth is a process of more coherently inclusive taking into account, and they move in the opposite direction, reason is displaced by fallacious machinations without few or none noticing it. A paradigm shift in rational and scientific validity has become the first imperative of our condition.

\section{REFERENCES}


Arrow, Kenneth (1963). Social Choice and Individual Values, New York: Wiley. Buchbinder, Howard and Newson, Janice (1991) "Social Knowledge and Market Knowledge", Gannet Centre Journal Spring/Summer, 17-30.

Alison Katz (2008), "Chernobyl: The Great Cover-Up”, Le Monde Diplomatique, April 2008, 13. Diamond, Jared (2005). Collapse: How Societies Choose to Fail or Succeed, New York: Viking. Edgeworth, Frances (1881[1932]). Mathematical Psychics, London: London School of Economics. Epstein, Samuel (2005). Cancer-Gate, New York: Baywood Press. Ferrie, Helkie (2008), "European Union leading the way", CCPA Monitor, March, 2008, 1,6. Gauthier, David (1986). Morals by Agreement, Oxford: York: Oxford University Press. Hodgson, Bernard (2001. Economics as Moral Science, Heidelberg: Springer. International Forum on Globalization (IFG) (1998). The Siena Declaration On the Crisis of Economic Globalization, Siena, Italy, September, 1998.

Meadows, Donella L., Meadows, Dennis L, Randers Jørgen, Behrens III, William H. (1972). The Limits to Growth: A Report for the Club of Rome's Project on the Predicament of Mankind, New York: Universe Books.

Miller, Peter and Westra, Laura, eds (2002). Just Ecological Integrity: The Ethics of Planetary Life, Boston: Rowman and Littlefield.

Marx, Karl (1859). Preface to An Introduction to the Critique of Political Economy in Marx and Engels Collected Works, Volume29, New York: International Publishers.

Marx, Karl (1867). Capital Volume 1, in Marx and Engels Collected Works, New York: International Publishers.

Mirowski, Philip (2000). Machine Dreams, Cambridge: Cambridge University Press. McMurtry, John (1998). Unequal Freedoms: The Global Market as an Ethical System. Toronto and Westport CT: Garamond Press and Kumarian Press.

McMurtry, John (1999). The Cancer Stage of Capitalism, London: Pluto Press. McMurtry, John (2000). "AT the Edge of a New Dark Age: The Corporate Takeover of Higher Research and Education", Economic Reform January 2000, 14-16.

McMurtry, John (2002). Value Wars: The Global Market versus the Life Economy, London: Pluto Press.

McMurtry, John (2007). "The Postmodern Voice of Empire: The Metalogic of Unaccountability" in Revathi Krishnaswamy and John Hawley, Minneapolis: University of Minesotta pp. 228-238. McMurtry, John (ed). Philosophy and World Problems, Paris-Oxford: Encyclopedia of Life Support Systems (EOLSS), UNESCO.

Pareto, Vilfredo, (1971 [1906]).Manual of Political Economy, New York: A.M. Kelley. Press, Eyal and Washburn, Jennifer (2000), “The Kept University”, Atlantic Monthly March 2000, 2-13.

Rawls, John (1971). A Theory of Justice, Cambridge Mass: Harvard University Press. Sen, Amartya (1977). "Rational Fools: A Critique of the Behavioral Foundations of Economic Theory", Philosophy and Public Affairs, 6, 317-44.

Sen, Amartya (1998). "The Possibility of Social Choice”, Nobel Lecture, December 8, 1998, 
published in American Economic Review, 89 (July 1999).

Sumner, Jennifer (2005). Sustainability and the Civil Commons: Rural Society in the Age of Globalization, Toronto: University of Toronto Press.

Turk, James, ed (2000). The Corporate Campus: Commercialization and the Dangers to Canada's Colleges and Universities: Toronto, Lorimer.

Weisbrot, M., Baker, D., and Rosnick, D. (2006). "The Scorecard on Development: 25 Years of Diminishing Progress", International Journal of Health Services 36,2: 211-234.

World Commission of the Environment and Development (1986). Our Common Future, New York: Oxford University Press. 